\title{
Imaging strategies for detection of urgent conditions in patients with acute abdominal pain: diagnostic accuracy study
}

\author{
Wytze Laméris, research fellow ,,2 Adrienne van Randen, research fellow, ${ }^{2,1} \mathrm{H}$ Wouter van Es, consultant \\ radiologist, ${ }^{3}$ Johannes P M van Heesewijk, consultant radiologist, ${ }^{3}$ Bert van Ramshorst, consultant surgeon, ${ }^{4}$ \\ Wim H Bouma, consultant surgeon, ${ }^{5}$ Wim ten Hove, consultant radiologist, ${ }^{6}$ Maarten S van Leeuwen, \\ consultant radiologist, ${ }^{7}$ Esteban M van Keulen, consultant radiologist, ${ }^{8}$ Marcel G W Dijkgraaf, methodologist, ${ }^{9}$ \\ Patrick M M Bossuyt, professor of clinical epidemiology, ${ }^{9}$ Marja A Boermeester, consultant surgeon and \\ clinical epidemiologist, ${ }^{1}$ Jaap Stoker, professor of radiology² on behalf of the OPTIMA study group
}

${ }^{1}$ Department of Surgery, Academic Medical Center, Amsterdam, Netherlands

${ }^{2}$ Department of Radiology, Academic Medical Center, Meibergdreef 9, $1105 \mathrm{AZ}$ Amsterdam

${ }^{3}$ Department of Radiology, St Antonius Hospital, Nieuwegein, Netherlands

${ }^{4}$ Department of Surgery, St Antonius Hospital, Nieuwegein

${ }^{5}$ Department of Surgery, Gelre Hospitals, Apeldoorn, Netherlands ${ }^{6}$ Department of Radiology, Gelre Hospitals, Apeldoorn

${ }^{7}$ Department of Radiology, University Medical Center Utrecht, Utrecht, Netherlands

${ }^{8}$ Department of Radiology, Tergooi Hospitals, Hilversum, Netherlands

${ }^{9}$ Department of Clinical Epidemiology, Biostatistics, and Bioinformatics, Academic Medical Center, Amsterdam

Correspondence to: J Stoker

j.stoker@amc.uva.nl

Cite this as: $B M J$ 2009;339:b2431 doi:10.1136/bmj.b2431

\section{ABSTRACT}

Objective To identify an optimal imaging strategy for the accurate detection of urgent conditions in patients with acute abdominal pain.

Design Fully paired multicentre diagnostic accuracy study with prospective data collection.

Setting Emergency departments of two university hospitals and four large teaching hospitals in the Netherlands.

Participants 1021 patients with non-traumatic abdominal pain of $>2$ hours' and $<5$ days' duration. Exclusion criteria were discharge from the emergency department with no imaging considered warranted by the treating physician, pregnancy, and haemorrhagic shock.

Intervention All patients had plain radiographs (upright chest and supine abdominal), ultrasonography, and computed tomography (CT) after clinical and laboratory examination. A panel of experienced physicians assigned a final diagnosis after six months and classified the condition as urgent or non-urgent.

Main outcome measures Sensitivity and specificity for urgent conditions, percentage of missed cases and false positives, and exposure to radiation for single imaging strategies, conditional imaging strategies (CT after initial ultrasonography), and strategies driven by body mass index and age or by location of pain.

Results 661 (65\%) patients had a final diagnosis classified as urgent. The initial clinical diagnosis resulted in many false positive urgent diagnoses, which were significantly reduced after ultrasonography or CT. CT detected more urgent diagnoses than did ultrasonography: sensitivity was $89 \%$ (95\% confidence interval $87 \%$ to $92 \%$ ) for CT and $70 \%$ (67\% to $74 \%$ ) for ultrasonography ( $\mathrm{P}<0.001)$. A conditional strategy with CT only after negative or inconclusive ultrasonography yielded the highest sensitivity, missing only $6 \%$ of urgent cases. With this strategy, only $49 \%$ (46\% to $52 \%$ ) of patients would have CT. Alternative strategies guided by body mass index, age, or location of the pain would all result in a loss of sensitivity.

Conclusion Although CT is the most sensitive imaging investigation for detecting urgent conditions in patients with abdominal pain, using ultrasonography first and CT only in those with negative or inconclusive ultrasonography results in the best sensitivity and lowers exposure to radiation.

\section{INTRODUCTION}

Acute abdominal pain accounts for 5-10\% of visits to the emergency department. Rapid and accurate detection of urgent conditions is vital for managing patients and for efficient throughput of patients. Decision making in patients with abdominal pain on the basis of clinical and laboratory evaluation alone can result in unnecessary interventions or in delayed treatment of urgent conditions. Several forms of imaging, of which ultrasonography and computed tomography (CT) are the most often used, can assist in clinical decision making. Imaging has been shown to have a positive effect on the accuracy of the clinical diagnosis, ${ }^{12}$ to lead to changes in decisions about management, ${ }^{34}$ and to increase the level of diagnostic certainty in patients with acute abdominal pain. ${ }^{56}$ The use of early CT in patients with abdominal pain has been shown to reduce the number of serious diagnoses missed. ${ }^{7}$

Over the years, diagnostic imaging in the emergency department has been responsible for an increase in hospital costs, ${ }^{89}$ and CT has been a growing source of exposure to radiation in adult patients. ${ }^{10}$ This calls for a rational, evidence based approach to imaging in patients with abdominal pain. The American College of Radiology has made available guidelines for imaging in patients with abdominal pain and specific locations of abdominal tenderness. ${ }^{11-14}$ This differentiation of use of imaging with patient tailored guidelines may not be sufficient to cover the wide spectrum of clinical presentations of abdominal pain. 
An optimal imaging strategy in patients with abdominal pain would lead to timely identification of urgent conditions, without exposing patients to unnecessary forms of imaging. Unfortunately, many of the studies on imaging for acute abdominal pain have evaluated investigations in isolation, and several show methodological shortcomings such as retrospective collection of data or differential verification of test results.

We evaluated the added value of plain radiographs, ultrasonography, and computed tomography after clinical evaluation for making urgent diagnoses in patients presenting with abdominal pain. We compared the accuracy and costs of 11 diagnostic strategies, by using data systematically collected in a consecutive series of unselected patients presenting with acute, non-traumatic abdominal pain at the emergency department, all of whom were investigated with a structured overcomplete imaging protocol.

\section{METHODS}

We collected data prospectively within a multicentre, fully paired diagnostic accuracy study investigating the added value of radiological investigations after clinical assessment in patients presenting with acute abdominal pain. ${ }^{15}$ Eligible patients were adults $(\geq 18$ years $)$ presenting with non-traumatic abdominal pain of more than two hours' and less than five days' duration. Patients were identified at the emergency department and were either self referred or referred by their general practitioner. Recently discharged patients admitted for reasons other than abdominal pain but who developed abdominal pain were also eligible, as were postoperative patients after a pain-free period. We excluded patients in haemorrhagic shock resulting from gastrointestinal bleeding or ruptured aortic aneurysm, as well as pregnant women. Patients who were to be discharged from the emergency department without imaging being considered to be warranted by the treating physician were not invited into the study.

Six hospitals participated in the inclusion of patients: two university hospitals (Academic Medical Center Amsterdam and University Medical Center Utrecht) and four large teaching hospitals (Antonius Hospital Nieuwegein, Gelre Hospital Apeldoorn, Tergooi Hospital Hilversum, and Onze Lieve Vrouwe Gasthuis Amsterdam). The Onze Lieve Vrouwe Gasthuis and Tergooi Hospital included patients from Monday to Friday between 9 am and 5 pm. In all other hospitals, patients were included seven days a week between 8 am and $11 \mathrm{pm}$.

Data were recorded prospectively on a digital case record form. The study coordinators continuously monitored the quality and completeness of data. Automatic registration of the time of data entry allowed monitoring of prospective real time data recording by treating physicians and radiologists.

\section{Diagnostic protocol and observer experience}

Eligible patients gave written informed consent before inclusion. Figure 1 shows a flow chart of the study protocol. Included patients had a medical history, physical examination, and initial laboratory investigations. A diagnosis based on clinical evaluation and laboratory investigation was recorded. Thereafter, patients were investigated with a full structured imaging protocol, including upright chest and supine abdominal plain radiography, abdominal ultrasonography, and CT. After the physician in the emergency department had assessed the plain radiographs, a new diagnosis was recorded. Ultrasonography and CT were each read without disclosure of any results of the other investigation, but clinical information was available to the readers of ultrasonography and CT. The reader recorded the radiological diagnosis from the same list of diagnoses that was used by the physicians in the emergency department.

A general survey of the abdomen was done with ultrasonography, using the graded compression technique. ${ }^{16} \mathrm{CT}$ images were obtained from the diaphragm to the groins. CT protocols were based on the following protocol: effective mAs level of 165 , $120 \mathrm{kV}$, maximum $2.5 \mathrm{~mm}$ collimation, maximum $3 \mathrm{~mm}$ slice width, and $0.5 \mathrm{~s}$ rotation time, and with injection of $125 \mathrm{ml}$ intravenous contrast after a 60 second delay with $3 \mathrm{ml} / \mathrm{s}$; no oral or rectal contrast agents were used. CT done after office hours was reevaluated blinded the next day by a radiologist.

\section{Final diagnosis}

We followed all included patients for at least six months. We systematically collected data on clinical, laboratory, and surgical findings; pathology results; imaging reports; and outcomes of treatment. We

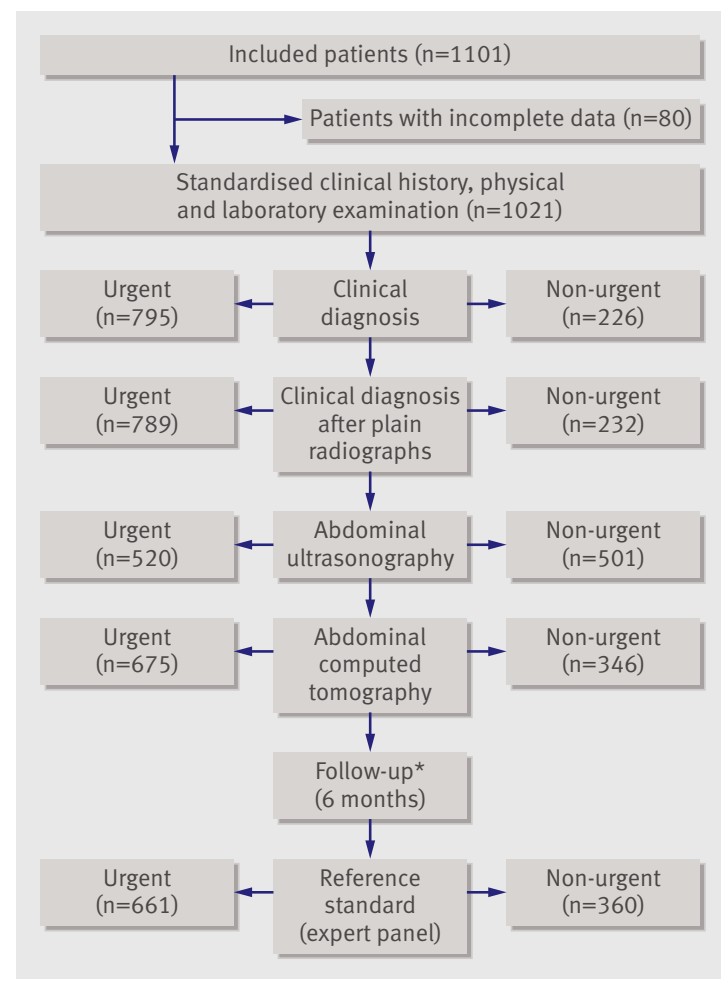

Fig 1| Flow chart of study protocol. *Completed for all 1021 patients 
contacted general practitioners to verify patients' outcomes. An expert panel formed of two gastrointestinal surgeons and an abdominal radiologist with long term clinical experience assigned a final diagnosis (panel members are listed in the web appendix). The panel members had not been involved in the investigation or management of the evaluated cases. Each panel member individually evaluated every case; data were presented in a standardised format, including all available information collected during follow-up. Disagreements on the final diagnosis were resolved during consensus meetings. All final diagnoses were classified as urgent or non-urgent. We defined urgent diagnoses as conditions needing treatment within 24 hours.

\section{Diagnostic strategies}

The fully paired study design, with all imaging tests in all patients and with the panel based final diagnosis as the reference standard, allowed a comparison of the diagnostic accuracy of multiple imaging strategies. We compared the following single imaging strategies: (1) diagnosis after clinical evaluation, (2) clinical diagnosis plus plain radiographs, (3) ultrasonography in all patients, and (4) CT in all patients. In addition, we investigated the following conditional ultrasonography and CT strategies: (5) ultrasonography first in all patients and CT in those with a negative or inconclusive ultrasonography and (6) ultrasonography in all patients and CT only in patients with inconclusive ultrasonography.

We also investigated imaging strategies driven by patients' characteristics, in which only selected patients would have initial ultrasonography, with CT after a negative or inconclusive ultrasonography, whereas all others would have initial CT. For initial ultrasonography in these strategies, we selected (7) younger patients $(<45$ years), for whom exposure to ionising radiation is most hazardous, and (8) nonobese patients (body mass index $<30 \mathrm{~kg} / \mathrm{m}^{2}$ ), in whom ultrasonography is more likely to be diagnostic. In another strategy (9) both younger and non-obese patients would have initial ultrasonography.

A common clinical view is that the choice of imaging for acute abdominal pain should be guided by the location of pain. Therefore, we investigated strategies in which the selection of ultrasonography or CT was driven by the abdominal quadrant in which the pain was predominantly located. In strategy (10) the choice of investigation was based on the American College of Radiology guidelines, which recommend ultrasonography for pain in the right upper quadrant and computed tomography for pain in the other quadrants and for diffuse abdominal pain. ${ }^{11-14} \mathrm{In}$ an alternative pain location driven strategy (11) patients with pain in the right upper quadrant or right lower quadrant would have initial ultrasonography and all others would have initial CT. The box summarises the strategies investigated.

Data analysis

We calculated sensitivity and specificity for detecting urgent conditions for each of the 11 diagnostic strategies, by comparing the results of the strategy with the final diagnosis assigned by the expert panel. We also calculated the percentage of missed cases (1 -sensitivity) and the percentage of false positives (false positives/all positives). We investigated the stability of the performance of ultrasonography by comparing the accuracy results between radiologists, between supervised and non-supervised residents, and between the participating centres. We plotted the accuracy of all strategies in a receiver operating characteristics space. For the calculation of diagnostic accuracy, we treated inconclusive test results as negative. We considered results of imaging to be inconclusive if no radiological diagnosis was made and abnormalities were not excluded on imaging.

We used the McNemar test statistic for comparing sensitivities and specificities between single imaging strategies. We compared the clinical diagnosis and the most accurate single test strategy with the multiinvestigation strategies. We evaluated the gain in accuracy in strategies from combining two forms of imaging relative to the most accurate single test strategy by direct comparison of the corresponding positive and negative likelihood ratios of the strategies, as described by Macaskill and colleagues. ${ }^{17} \mathrm{We}$ calculated the percentage of patients who had ultrasonography and the percentage who were exposed to CT related ionising radiation for each strategy.

\section{RESULTS}

Inclusion started in March 2005, and 1101 patients were included over the following 21 months. For six months we recorded invited patients who declined in the Academic Medical Centre, Amsterdam; 2\% (4/ 188) of the patients approached refused to participate. Data collection could not be completed for 80 patients. We found no significant differences between these 80 patients and the included patients in terms of age, sex, or time or type of presentation. The mean age of the remaining 1021 patients was 47 (range 19-94) years; $55 \%(\mathrm{n}=565)$ were female. Most $(75 \% ; \mathrm{n}=766)$ patients had been referred to the emergency department by a general practitioner; 17\% (169) were self referrals, 7\% (73) were referred by other medical specialties, and 1\% (13) were presented by emergency services.

Surgical residents evaluated $74 \%(n=757)$ of patients, and emergency medicine residents evaluated the other $26 \%$ (264). The mean clinical experience of the residents was 25 months (range 2 months to 8.7 years). The ultrasonography was done by a radiological resident in 57\% (582) of patients and by a staff radiologist in $43 \%$ (439). Fifty two per cent (300/582) of the ultrasonography examinations by residents were done during office hours under the supervision of a staff radiologist, and $48 \%$ (282) were done after office hours without supervision. The experience of the ultrasonography and CT readers ranged from one year's residency to more than 30 years' experience as a radiologist. Of the 54 radiological residents, $50 \%(n=27)$ were first or second year residents and 50\% (27) were third to fifth year residents. Of the 46 radiologists, $26 \%$ 
The 11 imaging strategies investigated

1) Clinical diagnosis

Single test strategies

2) Clinical diagnosis after plain radiographs

3) Ultrasonography in all patients

4) Computed tomography (CT) in all patients

Conditional strategies

5) Ultrasonography in all patients; CT if ultrasonography negative or inconclusive

6) Ultrasonography in all patients; CT if ultrasonography inconclusive

Strategies driven by patients' characteristics

7) If age $<45$ then ultrasonography and $\mathrm{CT}$ if ultrasonography negative or inconclusive; if age $\geq 45$ then $C T$

8) If body mass index $<30$ then ultrasonography and CT if ultrasonography negative or inconclusive; if body mass index $\geq 30$ then $\mathrm{CT}$

9) If body mass index $<30$ or age $<45$ then ultrasonography and CT if ultrasonography negative or inconclusive; CT in all other patients

\section{Strategies driven by location of pain}

10) If tenderness in right upper quadrant then ultrasonography; if tenderness in right lower quadrant, left upper quadrant, or left lower quadrant then $\mathrm{CT}$; if diffuse tenderness then $\mathrm{CT}$; $\mathrm{CT}$ in all other patients

11) If tenderness in right upper quadrant or right lower quadrant then ultrasonography; if tenderness in left lower quadrant or left upper quadrant then $\mathrm{CT}$; if diffuse tenderness then $\mathrm{CT}$; $\mathrm{CT}$ in all other patients specialists $(53 \% ; 39 / 73)$, or emergency services $(54 \%$; 7/13) $\left(\chi^{2} \mathrm{P}=0.009\right)$.

\section{Diagnostic accuracy}

Table 2 shows the accuracy results for each strategy. Figure 3 shows these results plotted in a receiver operating characteristics space (see web appendix for more information on the receiver operating characteristics space). The clinical diagnosis with or without plain radiographs had a high sensitivity but lacked specificity for urgent cases. Compared with the clinical diagnosis, the use of ultrasonography in all patients (ultrasonography strategy) reduced the number of false positive urgent diagnoses, but 30\% of urgent conditions would still be missed. Ultrasonography as a single test was inferior to CT as a single test (CT strategy) for the detection of urgent conditions, as the sensitivity was significantly higher for CT (89\%) than for ultrasonography $(70 \%, \mathrm{P}<0.001)$. The sensitivities of both strategies were not significantly higher than with clinical evaluation alone; only specificities were significantly improved.

The performance of ultrasonography was stable between the participating centres. The sensitivity of $65 \%$ (95\% confidence interval $58 \%$ to $72 \%$ ) for ultrasonography done by residents without supervision after working hours was significantly lower than sensitivity of $74 \%(69 \%$ to $74 \%)$ for ultrasonography by radiologists $(\mathrm{P}=0.03)$. The sensitivity was $69 \%(82 \%$ to $91 \%$ ) for ultrasonography done by residents under supervision, which was not significantly lower than for ultrasonography done by radiologists $(\mathrm{P}=0.20)$. The specificity for urgent conditions did not differ significantly between radiologists and supervised or unsupervised residents $(\mathrm{P}=0.70)$.

Application of CT after a negative or inconclusive ultrasonography result (strategy 5) had a higher sensitivity than the clinical diagnosis without imaging: 94\% versus $88 \%(\mathrm{P}<0.001)$. This conditional strategy would result in a significantly lower number of missed urgent conditions compared with CT only: $6 \%$ versus $11 \%$, with a higher sensitivity $(\mathrm{P}<0.001)$ but a lower specificity $(\mathrm{P}<0.001)$. An alternative conditional strategy with CT only after inconclusive ultrasonography (strategy 6) would further reduce use of CT (27\% of patients) but would increase the proportion of missed urgent conditions from $6 \%$ to $15 \%$.

Five hundred and twelve $(50 \%)$ patients were older than 45 , and $157(15 \%)$ patients had a body mass index exceeding 30. Nine per cent of urgent conditions would be missed with a strategy in which imaging is based on body mass index (strategy 8) compared with $10 \%$ for the strategies based on age (strategy 7) or on both age and body mass index (strategy 9 ). The diagnostic accuracy of the strategy reflecting the American College of Radiology guidelines (10) was comparable to that of the CT only strategy. The use of ultrasonography for both pain in the right upper quadrant and pain in the right lower quadrant (strategy 11) would lead to $16 \%$ missed urgent conditions. 


\section{Exposure to radiation}

Table 2 shows use of ultrasonography and CT for each strategy. CT related exposure to radiation would vary between $56 \%$ and $81 \%$ for the strategies driven by patients' characteristics and between 65\% and 95\% for the strategies driven by location of pain. The second lowest use of CT (49\%) would be achieved with the conditional CT strategy, the strategy that also had the highest sensitivity in detecting urgent diagnoses.

\section{DISCUSSION}

In this study, relying on the clinical diagnosis would have led to a high number of false positive urgent diagnoses, whereas the use of ultrasonography as the only imaging investigation would have given an unacceptably high number of missed urgent conditions. In comparison, computed tomography after clinical evaluation had better accuracy. The use of CT conditional on a negative or inconclusive ultrasonography result had the highest sensitivity for urgent conditions and would result in the lowest overall exposure to radiation. Use of imaging driven by patients' characteristics or location of pain would have led to a loss in accuracy compared with the conditional CT strategy.

\section{Potential limitations}

We acknowledge several potential limitations of this analysis. The study design, with its full diagnostic investigation in all enrolled patients, carefully collected data, and a diagnosis assigned after each step, allowed us to make the comparisons presented here, without doing a randomised clinical trial with 11 groups. Our approach allowed a comparison of imaging strategies but no direct evaluation of the effects of imaging on patients' management and outcome. In this study, management after completion of

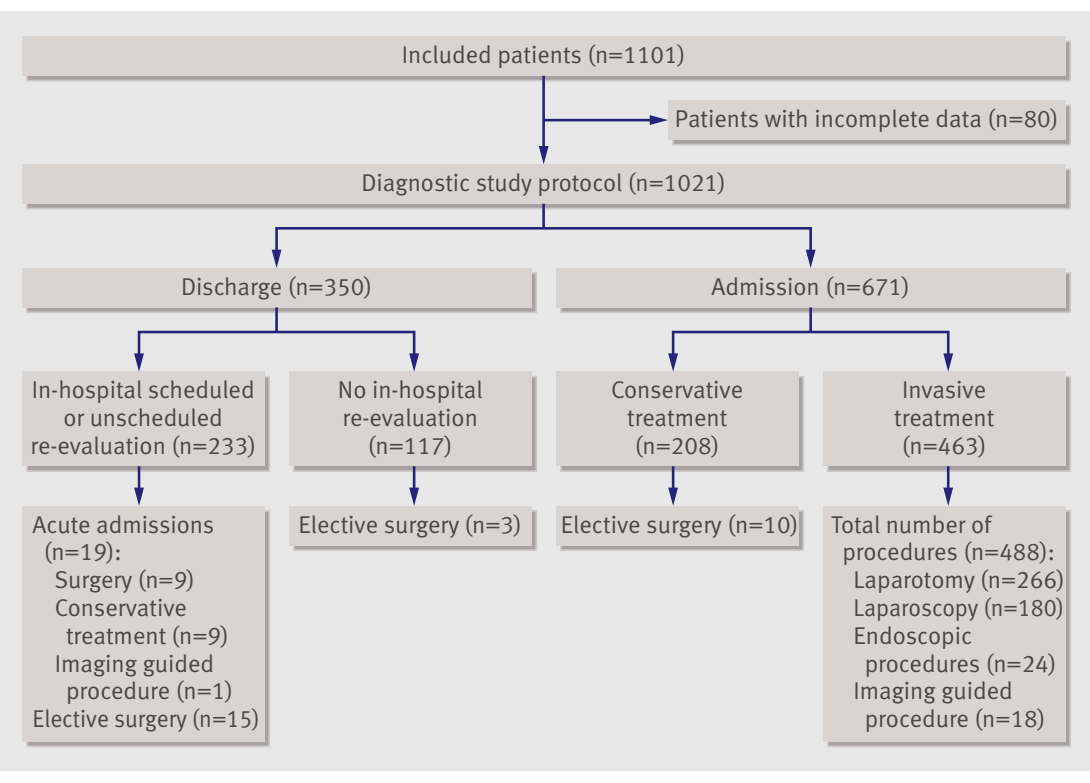

Fig 2 Management of patients after completion of the diagnostic protocol. *Laparoscopy converted to laparotomy in 26 patients
Table 1| Final diagnoses assigned by expert panel

\begin{tabular}{|c|c|}
\hline Final diagnoses in 1021 patients & No (\%) \\
\hline \multicolumn{2}{|l|}{ Urgent } \\
\hline Acute appendicitis & $284(28)$ \\
\hline Acute diverticulitis & $118(12)$ \\
\hline Bowel obstruction & $68(7)$ \\
\hline Acute cholecystitis & $52(5)$ \\
\hline Acute pancreatitis & $28(3)$ \\
\hline Gynaecological diseases* & $27(3)$ \\
\hline Urological diseases $†$ & $22(2)$ \\
\hline Abscessł & $14(1)$ \\
\hline Perforated viscus & $13(1)$ \\
\hline Bowel ischaemia & $12(1)$ \\
\hline Pneumonia & $11(1)$ \\
\hline Retroperitoneal or abdominal wall bleeding & $9(1)$ \\
\hline Acute peritonitis & $3(0.3)$ \\
\hline Total urgent diagnoses & $661(65)$ \\
\hline \multicolumn{2}{|l|}{ Non-urgent } \\
\hline Non-specific abdominal pain & $183(18)$ \\
\hline Gastrointestinal diseases§ & $56(5)$ \\
\hline Hepatic, pancreatic, and biliary diseases $\mathbb{~}$ & $43(4)$ \\
\hline Inflammatory bowel disease & $30(3)$ \\
\hline Urological diseases ${ }^{\star \star}$ & $20(2)$ \\
\hline Gynaecological diseases†† & $9(1)$ \\
\hline Malignancył‡ & $5(0.5)$ \\
\hline Hernia & $2(0.2)$ \\
\hline Other & $12(1)$ \\
\hline Total non-urgent diagnoses & $360(35)$ \\
\hline \multicolumn{2}{|c|}{$\begin{array}{l}\text { *Ovarian torsion, pelvic inflammatory disease, bleeding/ruptured ovarian } \\
\text { cyst. } \\
\text { †Renal and ureteral stones with obstruction, hydronephrosis, } \\
\text { pyelonephritis. } \\
\text { †Intra-abdominal abscess, retroperitoneal abscess, hepatic abscess, } \\
\text { tubo-ovarian abscess. } \\
\text { §Gastritis, gastroenteritis, peptic ulcer, acute epiploic appendagitis, } \\
\text { constipation. } \\
\text { ๆHepatic metastases, cholecystolithiasis, chronic pancreatitis. } \\
\text { ^* Renal and ureteral stones without obstruction, urinary tract infection. } \\
\text { ††Ovulation pain/bleeding, endometriosis, menstrual pain, uterine } \\
\text { myoma, benign adnexal cyst. } \\
\text { ††Pancreatic, gastrointestinal, and kidney malignancies. }\end{array}$} \\
\hline
\end{tabular}

the diagnostic protocol was always based on the result of all diagnostic tests.

This study had a pre-imaging selection of patients, which should be kept in mind when attempting to generalise the results. Most patients were referred to the emergency department by general practitioners, and patients discharged from the emergency department without imaging were not included. This selection resulted in a relatively high prevalence of urgent conditions. In other countries, the referral patterns, the threshold to attend the emergency department, and the threshold for imaging may differ. As a result, the prevalence and spectrum of urgent conditions could differ between settings, which should be kept in mind when applying our results to different settings.

We classified diagnoses as urgent or not before the start of the study. We defined urgent conditions as those needing treatment within 24 hours, making quick and accurate diagnosis important. Although we used a multimember panel, other clinical groups might 
classify these conditions and individual cases in a slightly different way.

Detection of urgent abdominal conditions

A high sensitivity for urgent conditions is clinically important, as patients with an urgent diagnosis being discharged home and left untreated is undesirable. False positive urgent diagnoses, on the other hand, could lead to overtreatment. In resource constrained settings, where access to ultrasonography and CT is limited, the percentage of missed cases is likely to be higher than with the conditional CT strategy described here, as we found that clinical examination had a lower sensitivity. The associated low specificity could lead to the overtreatment of many patients with non-urgent conditions.

The gain in diagnostic performance of the conditional CT strategy compared with the CT only strategy represents a trade-off between a gain in sensitivity and a loss in specificity. No disproportionate decrease in specificity accompanied the 5\% increase in sensitivity when switching from a general to a conditional CT strategy. Assessing whether the health benefits for patients being correctly identified with an urgent condition are larger than the harms of overtreatment associated with an incorrectly assigned urgent condition is beyond the scope of this study.

In clinical practice, physicians consciously and subconsciously distinguish categories of urgency for patients with acute abdominal pain in the emergency department. Apart from patients with a low clinical

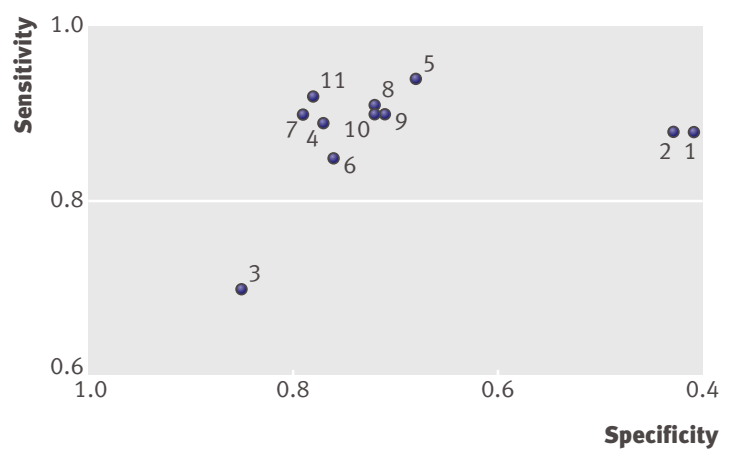

Fig 3| Diagnostic performance of all imaging strategies presented in receiver operating characteristics space. Numbers correspond to strategies in the box; strategy 5 is ultrasonography in all patients with computed tomography if ultrasonography is negative or inconclusive. See web appendix for more information

suspicion who were discharged from the emergency department without imaging, all other eligible consecutive patients were invited to enter the study. As a consequence, we also included patients in whom the diagnosis after clinical evaluation was clear, with limited added value of imaging. In other patients, urgent diagnoses were suspected after clinical evaluation but could not be made with high accuracy and certainty. This category of patients, in which imaging has the largest added value after clinical evaluation, probably comprises the vast majority of included patients.

Table 2 | Diagnostic accuracy and use of imaging for each imaging strategy. Values are percentages (95\% confidence intervals); numbers

\begin{tabular}{|c|c|c|c|c|c|c|}
\hline Imaging strategies & $\begin{array}{l}\text { Sensitivity (true } \\
\text { positives) }\end{array}$ & $\begin{array}{c}\text { Specificity } \\
\text { (true negatives) }\end{array}$ & $\begin{array}{l}\text { Missed urgent } \\
\text { diagnoses } \\
\text { (false negatives) }\end{array}$ & False positives* & CT use & US use \\
\hline 1) Clinical diagnosis & 88 (86 to 91); 582 & 41 (36 to 46$) ; 147$ & $12(79)$ & $27 ; 213 / 795$ & 0 & 0 \\
\hline \multicolumn{7}{|l|}{ Single imaging strategies } \\
\hline 2) Clinical diagnosis after plain radiographs & 88 (86 to 91); 583 & 43 (38 to 48 ); 154 & $12(78)$ & $26 ; 206 / 789$ & 0 & 0 \\
\hline 3) Ultrasonography in all patients & 70 (67 to 74$) ; 465$ & 85 (81 to 88$) ; 305$ & $30(196)$ & $11 ; 55 / 520$ & 0 & $100 ; 1021$ \\
\hline 4) Computed tomography in all patients & 89 (87 to 92); 591 & 77 (72 to 81$) ; 276$ & $11(70)$ & $12 ; 84 / 675$ & $100 ; 1021$ & 0 \\
\hline \multicolumn{7}{|l|}{ Conditional strategies } \\
\hline 5) US in all patients; CT if US negative & 94 (92 to 96); 620 & 68 (64 to 73$) ; 246$ & $6(41)$ & $16 ; 114 / 734$ & 49 (46 to 52$) ; 501$ & $100 ; 1021$ \\
\hline 6) US in all patients; CT if US inconclusive & 85 (82 to 88$) ; 563$ & 76 (71 to 80$) ; 272$ & $15(98)$ & $14 ; 88 / 651$ & 27 (24 to 29$) ; 271$ & $100 ; 1021$ \\
\hline \multicolumn{7}{|l|}{ Strategies driven by patients' characteristics } \\
\hline $\begin{array}{l}\text { 7) If age }<45 \text { then US and CT if US negativet; if } \\
\text { age } \geq 45 \text { then CT }\end{array}$ & 90 (87 to 92); 593 & 72 (67 to 76$) ; 258$ & $10(68)$ & $15 ; 102 / 695$ & 78 (76 to 81$) ; 800$ & 47 (44 to 50$) ; 484$ \\
\hline $\begin{array}{l}\text { 8) If } \mathrm{BMI}<30 \text { then US and } \mathrm{CT} \text { if US negative }+ \text {; if } \\
\mathrm{BMI} \geq 30 \text { then } \mathrm{CT}\end{array}$ & 91 (88 to 93); 599 & 71 (67 to 76$) ; 257$ & $9(62)$ & $15 ; 103 / 702$ & 56 (53 to 59$) ; 570$ & 85 (82 to 87$) ; 864$ \\
\hline $\begin{array}{l}\text { 9) If } B M I<30 \text { or age }<45 \text { then US and CT if US } \\
\text { negativet; } C T \text { in all other patients }\end{array}$ & 90 (87 to 92); 593 & 72 (68 to 77$) ; 260$ & $10(68)$ & $14 ; 100 / 693$ & 81 (78 to 83$) ; 825$ & 42 (39 to 45$) ; 426$ \\
\hline \multicolumn{7}{|l|}{ Strategies driven by location of pain } \\
\hline $\begin{array}{l}\text { 10) If tenderness RUQ then US; if tenderness } \\
\text { RLQ, LUQ, or LLQ then CT; if diffuse tenderness } \\
\text { then CT; CT in all other patients }\end{array}$ & 89 (87 to 92); 591 & 78 (73 to 82); 279 & $11(70)$ & $12 ; 81 / 672$ & 95 (93 to 96$) ; 970$ & 5 (4 to 7$) ; 51$ \\
\hline $\begin{array}{l}\text { 11) If tenderness RUQ or RLQ then US; if } \\
\text { tenderness LLQ or LUQ then CT; if diffuse } \\
\text { tenderness then CT; CT in all other patients }\end{array}$ & 84 (81 to 87$) ; 555$ & 79 (75 to 83); 285 & $16(106)$ & $12 ; 75 / 630$ & 65 (62 to 68$) ; 660$ & 35 (32 to 38$) ; 361$ \\
\hline
\end{tabular}

BMI=body mass index; $C T=$ computed tomography; LLQ=left lower quadrant; LUQ=left upper quadrant; RLQ=right lower quadrant; RUQ=right upper quadrant; US=ultrasonography. ${ }^{*}$ Calculated as false positives/all positives.

tIncluding inconclusive ultrasonography. 


\section{Observers' experience}

Residents in surgery and emergency medicine made the clinical diagnosis in our study. Their accuracy may have been negatively affected by their limited clinical experience, but we are not aware of studies that have evaluated the influence of clinical experience on the accuracy of the clinical diagnosis in acute abdominal pain. In our analysis, we found no effect of seniority of residents on clinical accuracy. The research setting, with standardised feedback on clinical diagnoses corrected by subsequent ultrasonography or CT, may also have influenced the accuracy of the clinical diagnosis.

We showed that the sensitivity of ultrasonography depended on observers' experience. Hertzberg et al previously showed a significant increase in the percentage of depicted anatomic landmarks on ultrasonography from 33\% after 50 ultrasonography examinations to $57 \%$ after 200 ultrasonography examinations by radiological residents during competence tests. ${ }^{18}$ The same effect was not shown for the diagnostic accuracy of ultrasonography, but a similar learning curve is likely. In our study, ultrasonography by residents without supervision resulted in a higher number of missed urgent conditions. In clinical practice with CT conditional on negative or inconclusive ultrasonography, secondary CT will more often be needed after ultrasonography done by unsupervised residents than after ultrasonography done by radiologists. The performance of CT is known to be less dependent on observers' experience in patients with acute abdominal pain. For acute diverticulitis, no significant difference in the accuracy of CT was found between a radiologist with 20 years' experience, a second year radiological resident, and a gastroenterologist. ${ }^{19}$ As all CT examinations in our study were read by a radiologist or under the supervision of a radiologist, a comparison of accuracy between residents and radiologists was not possible (nor was it needed).

\section{Implications for clinical practice}

For a diagnostic study to have impact in clinical practice, the results should be obtained in a representative population and setting. This multicentre study closely mimicked daily practice; a large number of residents

\section{WHAT IS ALREADY KNOWN ON THIS TOPIC}

Ultrasound and computed tomography increase the accuracy of the clinical diagnosis, increase diagnostic certainty, and influence management decisions in patients with acute abdominal pain

\section{WHAT THIS STUDY ADDS}

As a single imaging strategy, computed tomography is better overall than ultrasonography in detecting urgent conditions

A conditional computed tomography strategy, with ultrasonography in all patients and computed tomography only after negative or inconclusive ultrasonography, gives the highest sensitivity for detecting urgent conditions

With this conditional computed tomography strategy, only half of patients would require computed tomography and radiological observers, with varying levels of experience, participated. The multicentre setting included university and teaching hospitals. We believe that our results reflect the performance of imaging strategies in daily practice and are relevant to physicians managing patients with acute abdominal pain.

The use of imaging has been widely investigated for the two most common causes of acute abdominal pain - acute appendicitis and diverticulitis. In patients with suspected diverticulitis, imaging is helpful in detecting complications of diverticulitis to assist in therapeutic decisions. ${ }^{20}$ For suspected acute appendicitis, extensive use of CT has been shown to reduce rates of negative appendicectomy and lower overall hospital costs by preventing unnecessary admissions and surgical explorations. ${ }^{21-24}$ Although imaging in general showed a positive effect on patients' outcomes in acute appendicitis, the ideal diagnostic strategy for acute appendicitis remains a matter of debate, as imaging might not be necessary for certain presentations.

In unselected patients with acute abdominal pain, studies have shown that the use of diagnostic imaging positively affects the clinical diagnosis and influences decisions on management. ${ }^{136725-28}$ Diagnostic laparoscopy has also been shown to be an accurate investigation in patients with acute abdominal pain. ${ }^{2930}$ However, an invasive procedure can often be prevented with an accurate imaging strategy, as imaging reduced false positive clinical diagnoses. Considering the costs and invasiveness of diagnostic laparoscopy in comparison with imaging, this investigation should probably be reserved for clinically ill patients with non-diagnostic imaging or for therapeutic indications.

The lifetime risk of radiation induced fatal cancer is age dependent. The dose of radiation associated with abdominal CT in an acute abdomen is approximately $10 \mathrm{mSv}$. For a 25 year old patient, the estimated risk of induction of cancer for such a CT examination is about 1 in 900. The estimated risk of induction of fatal cancer is about 1 in $1800 .^{3132}$ For a 50 year old, the equivalent risks are 1 in 1500 and 1 in 2500. In general, consensus exists that the information obtained with diagnostic CT outweighs the risk associated with radiation and that the risk of induction of cancer should be seen in the light of the lifetime risk of cancer. In the future, magnetic resonance imaging may be valuable for making a diagnosis in acute abdominal pain. ${ }^{33}$ Until now, the availability of magnetic resonance imaging at the emergency department has been low and this investigation is mainly reserved for patients with a contraindication to $\mathrm{CT}$, such as pregnant patients.

\section{Conclusions}

Although imaging is widely applied in patients presenting with acute abdominal pain, imaging strategies had not previously been compared in a large prospective study of diagnostic accuracy. In this study, computed tomography led to the largest increase in accuracy after clinical evaluation, but a conditional strategy with CT after negative or inconclusive ultrasonography resulted in the highest overall sensitivity, with only 
$6 \%$ missed urgent conditions, and the lowest overall exposure to radiation. We therefore recommend use of ultrasonography as the initial investigation in the diagnostic investigation of patients presenting with acute abdominal pain, with $\mathrm{CT}$ after negative or inconclusive ultrasonography.

Contributors: MAB, JS, and PMMB initiated and designed the study, obtained funding, and were involved in analysing and interpreting data and in writing the manuscript. MGWD was involved in analysing and interpreting data, revising the manuscript, and economic evaluation. WL and AvR were responsible for data collection and were involved in analysing and interpreting data and in writing the manuscript. HWvE, JPMvH, BvR, WHB, WtH, MSvL, and EMvK were involved in analysing and interpreting data and in revising the manuscript. HWvE, WHB, WtH, and EMvK were expert panel members. JS is the guarantor. Study group members are listed in the web appendix.

Funding: The Dutch Organization for Health Research and Development (ZonMw), Health Care Efficiency Research programme, funded the study (ZonMw grant number: 945-04-308). ZonMw approved the study protocol after consulting national and international independent reviewers. The collection, analysis, and interpretation of data; writing of the article; and decision to submit were left to the authors' discretion and were not influenced by ZonMw.

Competing interests: None declared.

Ethical approval: The independent medical ethics committee of the Academic Medical Center Amsterdam approved the final study protocol. The medical ethics committees of the other five hospitals also gave thei approval after assessing the local feasibility of the study

1 Walsh PF, Crawford D, Crossling F, Sutherland GR, Negrette JJ, Shand J. The value of immediate ultrasound in acute abdominal conditions: a critical appraisal. Clin Radiol 1990;42:47-9.

2 Allemann F, Cassina P, Rothlin M, Largiader F. Ultrasound scans don by surgeons for patients with acute abdominal pain: a prospective study. Eur / Surg 1999;165:966-70.

3 Rosen MP, Siewert B, Sands DZ, Bromberg R, Edlow J, Raptopoulos V. Value of abdominal $\mathrm{CT}$ in the emergency department for patients with abdominal pain. Eur Radiol 2003;13:418-24.

4 Esses D, Birnbaum A, Bijur P, Shah S, Gleyzer A, Gallagher EJ. Ability of $\mathrm{CT}$ to alter decision making in elderly patients with acute abdominal pain. Am J Emerg Med 2004;22:270-2.

5 Bassler D, Snoey ER, Kim J. Goal-directed abdominal ultrasonography: impact on real-time decision making in the emergency department J Emerg Med 2003:24:375-8.

6 Sala E, Watson CJ, Beadsmoore C, Groot-Wassink T, Fanshawe TR, Smith JC, et al. A randomized, controlled trial of routine early abdominal computed tomography in patients presenting with nonspecific acute abdominal pain. Clin Radiol 2007;62:961-9.

$7 \mathrm{Ng}$ CS, Watson C), Palmer CR, See TC, Beharry NA, Housden BA, et al. Evaluation of early abdominopelvic computed tomography in patients with acute abdominal pain of unknown cause: prospective randomised study. BMJ 2002;325:1387.

8 Broder J, Warshauer DM. Increasing utilization of computed tomography in the adult emergency department, 2000-2005. Emerg Radiol 2006;13:25-30.

9 Mitka M. Costly surge in diagnostic imaging spurs debate. JAMA 2005;293:665-7.

10 Brenner DJ, Hall E). Computed tomography—an increasing source of radiation exposure. N Engl J Med 2007;357:2277-84.

11 Balfe DM, Levine MS, Ralls PW, Bree RL, DiSantis DJ, Glick SN, et al. Evaluation of left lower quadrant pain. American College of Radiology: ACR appropriateness criteria. Radiology 2000;215(suppl):167-71.
12 Bree RL, Ralls PW, Balfe DM, DiSantis DJ, Glick SN, Levine MS, et al. Evaluation of patients with acute right upper quadrant pain. American College of Radiology: ACR appropriateness criteria. Radiology 2000;215(suppl):153-7.

13 Ralls PW, Balfe DM, Bree RL, DiSantis DJ, Glick SN, Levine MS, et al Evaluation of acute right lower quadrant pain. American College of Radiology: ACR appropriateness criteria. Radiology 2000;215(suppl):159-66.

14 Saini S, Ralls PW, Balfe DM, Bree RL, DiSantis DJ, Glick SN, et al. Suspected abdominal abscess. American College of Radiology: ACR appropriateness criteria. Radiology 2000;215(suppl):173-9.

15 Lameris W, van Randen A, Dijkgraaf MG, Bossuyt PM, Stoker J, Boermeester MA. Optimization of diagnostic imaging use in patients with acute abdominal pain (OPTIMA): design and rationale. BMC Emerg Med 2007;7:9.

16 Puylaert JB, Rutgers PH, Lalisang RI, de Vries BC, van der Werf SD, Dorr IP, et al. A prospective study of ultrasonography in the diagnosis of appendicitis. N Engl J Med 1987;317:666-9.

17 Macaskill P, Walter SD, Irwig L, Franco EL. Assessing the gain in diagnostic performance when combining two diagnostic tests. Stat Med 2002;21:2527-46.

18 Hertzberg BS, Kliewer MA, Bowie JD, Carroll BA, DeLong DH, Gray L, et al. Physician training requirements in sonography: how many cases are needed for competence? AIR Am J Roentgenol 2000;174:1221-7.

19 Tack D, Bohy P, Perlot I, De Maertelaer V, Alkeilani O, Sourtzis S, et al Suspected acute colon diverticulitis: imaging with low-dose unenhanced multi-detector row CT. Radiology 2005;237:189-96

20 Pradel JA, Adell J-F, Taourel P, Djafari M, Monnin-Delhom E, Bruel J. Acute colonic diverticulitis: prospective comparative evaluation with US and CT. Radiology 1997;205:503-12.

21 Lee CC, Golub R, Singer AJ, Cantu R Jr, Levinson H. Routine versus selective abdominal computed tomography scan in the evaluation of right lower quadrant pain: a randomized controlled trial. Acad Emerg Med 2007;14:117-22

22 Walker S, Haun W, Clark J, McMillin K, Zeren F, Gilliland T. The value of limited computed tomography with rectal contrast in the diagnosis of acute appendicitis. Am / Surg 2000;180:450-4.

23 Raman SS, Osuagwu FC, Kadell B, Cryer H, Sayre J, Lu DS. Effect of CT on false positive diagnosis of appendicitis and perforation. $N$ Engl J Med 2008;358:972-3.

24 Rao PM, Rhea JT, Novelline RA, Mostafavi AA, McCabe CJ. Effect of computed tomography of the appendix on treatment of patients and use of hospital resources. N Engl J Med 1998;338:141-6.

25 Dhillon S, Halligan S, Goh V, Matravers P, Chambers A, Remedios D. The therapeutic impact of abdominal ultrasound in patients with acute abdominal symptoms. Clin Radiol 2002;57:268-71.

26 Williams RJ, Windsor AC, Rosin RD, Mann DV, Crofton M. Ultrasound scanning of the acute abdomen by surgeons in training. Ann $R$ Coll Surg Engl 1994;76:228-33.

27 Lewis LM, Klippel AP, Bavolek RA, Ross LM, Scherer TM, Banet GA. Quantifying the usefulness of $\mathrm{CT}$ in evaluating seniors with abdominal pain. Eur J Radiol 2007;61:290-6.

28 McGrath FP, Keeling F. The role of early sonography in the management of the acute abdomen. Clin Radiol 1991;44:172-4.

29 Majewski W. Diagnostic laparoscopy for the acute abdomen and trauma. Surg Endosc 2000;14:930-7.

30 Golash V, Willson PD. Early laparoscopy as a routine procedure in the management of acute abdominal pain: a review of 1,320 patients. Surg Endosc 2005; 19:882-5.

31 The 2007 recommendations of the International Commission on Radiological Protection: ICRP publication 103. Ann ICRP 2007:37(2-4):1-332.

32 Board on Radiation Effects Research (BRER). Health risks from exposure to low levels of ionizing radiation: BEIR VII phase 2 Washington DC: National Academies Press, 2006.

33 Stoker J. Magnetic resonance imaging and the acute abdomen. $\mathrm{Br}$ J Surg 2008;95:1193-4.

Accepted: 13 March 2009 